\title{
Incidencia y complicaciones del recién nacido gemelar
}

\author{
Incidence and complications of the twin newborn \\ Incidência e complicações do recém-nascido gêmeo
}

\begin{abstract}
Victor Apaza Maquera ${ }^{1 a}$
Manuel Ticona Rendón ${ }^{2 b, d}$

Diana Huanco Apaza ${ }^{2 c, d}$
\end{abstract}

(D) https://orcid.org/0000-0002-4819-1251
(D) https://orcid.org/0000-0001-9687-8384

\begin{abstract}
Resumen
Objetivo: Determinar la incidencia y complicaciones del recién nacido gemelar en un hospital público de Tacna, Perú. Material y métodos: Estudio descriptivo y retrospectivo en 472 recién nacidos vivos gemelos, se incluyó a todos los nacidos en el Hospital Hipólito Unanue de Tacna durante los años 2009-2018, con peso al nacer de 500 gramos a más. La información se obtuvo del Sistema Informático Perinatal (SIP), se presentan tablas y figuras descriptivas con frecuencias. Resultados: La incidencia de recién nacidos gemelares fue 1,39 por 100 nacidos vivos, con una discreta tendencia ascendente en los últimos 10 años. Las características perinatales más frecuentes fueron: nacido por cesárea (90,1\%), bajo peso al nacer (45,7\%), prematuro (53,2\%), Apgar menor de siete (7,7\%), y a los 5 minutos (3,01\%). Las complicaciones neonatales más frecuentes fueron: bajo peso al nacer (45,3\%), anemia (41,5\%), retardo en el crecimiento intrauterino (18,2\%), enfermedad de membrana hialina (11,1\%) y sepsis neonatal $(9,4 \%)$. La muerte neonatal representó el 4,95\% del total de recién nacidos gemelos. Conclusiones: La incidencia de gemelos en Tacna se encuentra en el promedio nacional e internacional, se caracteriza por haber nacido por cesárea, son de bajo peso y prematuros, las principales patologías fueron anemia, retardo de crecimiento intrauterino y membrana hialina, no se encontró diferencias entre el primer y segundo gemelo.
\end{abstract}

Palabras clave: embarazo múltiple, prematuridad, bajo peso al nacer, membrana hialina

\begin{abstract}
Objective: To determine the incidence and complications of the twin newborn in a public hospital in Tacna, Peru. Material and methods: Descriptive and retrospective study in 472 twin live newborns, all those born at the Hipólito Unanue Hospital in Tacna during the years 2009-2018 were included, with birth weights of 500 grams or more. The information was obtained from the Perinatal Computer System (SIP), tables and descriptive figures with frequencies are presented. Results: The incidence of twin newborns was 1.39 per 100 live births, with a slight upward trend in the last 10 years. The most frequent perinatal characteristics were: born by cesarean section (90.1\%), low birth weight (45.7\%), premature (53.2\%), Apgar less than seven (7.7\%), and at 5 minutes (3.01\%). The most frequent neonatal complications were: low birth weight $(45.3 \%)$, anemia $(41.5 \%)$, intrauterine growth delay $(18.2 \%)$, hyaline membrane disease $(11.1 \%)$ and neonatal sepsis $(9.4 \%)$. Neonatal death accounted for $4.95 \%$ of the total number of newborn twins. Conclusions: The incidence of twins in Tacna is in the national and international average, it is characterized by having been born by cesarean section, they are low weight and premature, the main pathologies were anemia, intrauterine growth retardation and hyaline membrane, no differences were found between the first and second twins.
\end{abstract}

Keywords: multiple pregnancy, prematurity, low birth weight, hyaline membrane

\footnotetext{
${ }^{1}$ Región de Salud Tacna. Centro de Salud Pocollay. Tacna, Perú

${ }^{2}$ Universidad Nacional Jorge Basadre. Tacna, Perú

${ }^{a}$ Médico cirujano

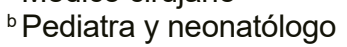

cObstetra

¿ Doctor en Salud Pública
} 


\begin{abstract}
Resumo
Objetivo: Determinar a incidência e complicações do recém-nascido gêmeo em um hospital público de Tacna, Peru. Material e métodos: Estudo descritivo e retrospectivo em 472 recém-nascidos vivos gêmeos, foram incluídos todos os nascidos no Hospital Hipólito Unanue de Tacna durante os anos 2009-2018, com peso ao nascer de 500 gramas a mais. A informação foi obtida do Sistema Informático Perinatal (SIP), apresenta-se tabelas e figuras descritivas com frequências. Resultados: A incidência de recém-nascidos gêmeos foi de 1,39 por 100 nascidos vivos, com uma discreta tendência ascendente nos últimos 10 anos. As características perinatais mais frequentes foram: nascido por cesariana $(90,1 \%)$, baixo peso à nascença $(45,7 \%)$, prematuro $(53,2 \%)$, Apgar inferior a sete $(7,7 \%)$ e 5 minutos $(3,01$ $\%)$. As complicações neonatais mais frequentes foram: baixo peso à nascença (45,3\%), anemia (41,5\%), atraso no crescimento intra-uterino (18,2\%), doença de membrana hialina $(11,1 \%)$ e sepsia neonatal $(9,4 \%)$. A morte neonatal representou 4,95 \% do total de recém-nascidos gêmeos. Conclusões: A incidência de gêmeos em Tacna se encontra na média nacional e internacional, caracteriza-se por ter nascido por cesariana, são de baixo peso e prematuros, as principais patologias foram anemia, retardo de crescimento intrauterino e membrana hialina, não foram encontradas diferenças entre o primeiro e o segundo gémeo.
\end{abstract}

Palavras-chave: gravidez múltipla, prematuridade, baixo peso ao nascer, membrana hialina

\section{Introducción}

Elembarazogemelaresunacondiciónanómala en el proceso de concepción humana. Su incidencia ha venido en ascenso de la mano de las técnicas de reproducción asistida, edad avanzada de la gestante, etc. En las últimas décadas en países desarrollados, se ha incrementado de 1 por 100 nacidos vivos a 1 por 70 nacidos vivos, con un aumento de un $40 \%$ en la tasa de embarazos gemelares y un aumento de 3 a 4 veces en embarazos múltiples de alto orden. Con ello, se incrementa también la probabilidad de obtener recién nacidos prematuros y con bajo peso para la edad gestacional. ${ }^{1}$

El embarazo gemelar está asociado a altas tasas de cualquier complicación posible del embarazo, con excepción de la macrosomía y el embarazo postérmino, incluyendo tanto complicaciones maternas como fetales. Este riesgo depende de su corionicidad mas no de su cigocidad. La complicación más común de un embarazo múltiple es que ocurra un parto prematuro. Más de la mitad de todos los mellizos nacen prematuramente. Los múltiples de orden superior casi siempre son prematuros. Además, el recién nacido de parto gemelar significa una situación delicada tanto para la madre como para el feto y es considerada una entidad patológica del proceso de reproducción. ${ }^{1}$

Desde el punto de vista neonatal, el embarazo gemelar contribuye de manera importante a la presencia de prematurez y bajo peso, con un incremento del 48 al $60 \%$ en los casos de prematuros, así como con una mayor morbilidad asociada, tanto en el recién nacido moderadamente prematuro (32 a 33,6 semanas) como en el prematuro tardío (34 a 36,6 semanas). ${ }^{2}$

El mayor riesgo de los embarazos múltiples es el parto prematuro. Se ha descrito un riesgo de parto prematuro de 5,4 veces más para embarazos gemelares, y de 9,4 veces más para embarazos triples. En Estados Unidos y Canadá, del 10 al $14 \%$ de los partos prematuros son atribuibles a embarazos gemelares. $^{3}$

La principal morbilidad encontrada en la población de gemelos dobles fue RCIU, probablemente asociada con otros determinantes maternos de bajo peso, como preeclampsia y probablemente ocasionada por una insuficiencia útero-placentaria, relacionada con una demanda metabólica mayor y una implantación anormal de la placenta, que caracteriza al embarazo múltiple. La RCIU representa el mayor factor de riesgo de morbilidad en los embarazos gemelares. Al comparar en un estudio las consecuencias, a largo plazo, entre los pequeños para edad gestacional versus los neonatos con peso apropiado para una edad gestacional de embarazos gemelares y triples, los pequeños para edad gestacional continuaron con bajo peso hasta los 3 y 6 años de edad. Además, presentaron una frecuencia más alta de problemas del lenguaje, alteraciones visuales y del desarrollo, por lo que sería de suma importancia conocer los resultados en el neurodesarrollo a largo plazo. ${ }^{4}$

Las anomalías congénitas contribuyen a empeorar el pronóstico en los embarazos gemelares, siendo 2 a 3 veces más comunes entre los gemelos monocigotos que en los embarazos únicos y dicigotos. En la mayoría de los casos sólo uno de los gemelos es afectado. La incidencia de malformaciones congénitas que encontramos estuvo entre 4,5 a $8,6 \% .^{5}$

El objetivo de este estudio fue conocer la incidencia y complicaciones neonatales del recién nacido gemelo del Hospital Hipólito Unanue de Tacna, durante el periodo 2009 a 2018. 


\section{Material y métodos}

Estudio descriptivo, retrospectivo y de corte transversal, realizado mediante el análisis de datos obtenidos a través de las historias clínicas del Sistema Informático Perinatal, durante el periodo enero 2009 a diciembre 2018, en el Hospital Hipólito Unanue de Tacna.

Se incluyó a la totalidad de 472 gemelos nacidos vivos, con peso al nacer de 500 gramos a más, nacidos en el hospital de estudio. La información de la base de datos se exportó al software SPSS versión 25. Para el análisis se utilizó tablas y figuras con estadística descriptiva como frecuencias.

\section{Resultados}

De los 34032 nacido vivos atendidos en el Hospital Hipólito Unanue de Tacna, durante los años 2009 a 2018, nacieron 472 gemelos, siendo la incidencia de 1,39 por 100 nacidos vivos, oscilando entre $1 \%$ en el año 2011 a 1,95 por 100 nacido vivos en el 2017 (Tabla 1), con una discreta tendencia ascendente en los diez años de estudio (Figura 1).

\section{Tabla 1}

Incidencia del recién nacido de parto gemelar

\section{Figura 1}

Incidencia y tendencia del recién nacido gemelar

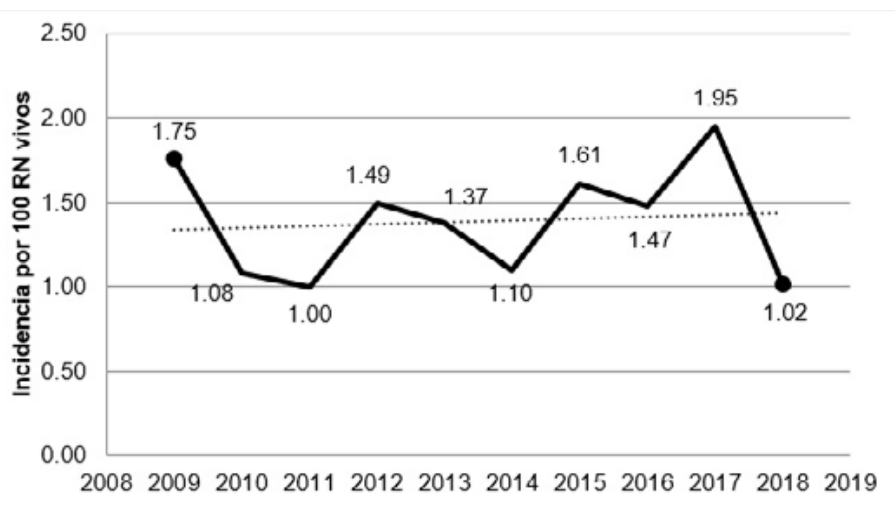

Los recién nacidos gemelos presentaron las siguientes características: el 50,86 \% del primer gemelo fue de sexo femenino y el $50,43 \%$ del segundo; nacieron por cesárea el $90,1 \%$; fueron de bajo peso el $45,7 \%$, siendo el $44 \%$ en el primero y el $45,7 \%$ en el segundo; pequeños para la edad gestacional el $14,66 \%$, en ambos gemelos; fueron prematuros, el $53,5 \%$ del primer gemelo y el $53,2 \%$ del segundo, con un promedio de edad gestacional de 35,5 semanas. Presentaron 7,3 \% de Apgar menor de siete al minuto en el primer gemelo y 7,7 $\%$ en el segundo gemelo, además de $3 \%$ de Apgar a los 5 minutos menor de siete (Tabla 2).

\begin{tabular}{lccc}
\hline Año de nacimiento & $\begin{array}{c}\text { Frecuencia de RN } \\
\text { gemerales }\end{array}$ & Total de nacimientos & Incidencia por 100 RNV \\
\hline 2009 & 58 & 3306 & 1,75 \\
2010 & 38 & 3510 & 1,08 \\
2011 & 34 & 3413 & 1 \\
2012 & 52 & 3482 & 1,49 \\
2013 & 46 & 3346 & 1,37 \\
2014 & 36 & 3275 & 1,10 \\
2015 & 56 & 3479 & 1,61 \\
2016 & 50 & 3392 & 1,47 \\
2017 & 68 & 3490 & 1,95 \\
2018 & 34 & 3339 & 1,02 \\
\hline Total & 472 & 34032 & 1,39 \\
\hline
\end{tabular}

Fuente: Sistema informático perinatal - HHUT 


\section{Tabla 2}

Características obstétricas y neonatales del recién nacido gemelar

\begin{tabular}{|c|c|c|c|c|c|c|c|}
\hline \multicolumn{2}{|c|}{ Número de gemelo } & \multicolumn{2}{|c|}{ Gemelo 1} & \multicolumn{2}{|c|}{ Gemelo 2} & \multicolumn{2}{|c|}{ Total } \\
\hline & & N. ${ }^{\circ}$ & $\%$ & N. ${ }^{\circ}$ & $\%$ & N. ${ }^{\circ}$ & $\%$ \\
\hline \multicolumn{2}{|l|}{ Total } & 232 & 100 & 232 & 100 & 464 & 100 \\
\hline \multirow{2}{*}{ Sexo } & Masculino & 114 & 49,14 & 115 & 49,57 & 229 & 49,35 \\
\hline & Femenino & 118 & 50,86 & 117 & 50,43 & 235 & 50,65 \\
\hline \multirow{2}{*}{ Terminación } & Cesárea & 209 & 90,09 & 209 & 90,09 & 418 & 90,09 \\
\hline & Espontáneo & 23 & 9,91 & 23 & 9,91 & 46 & 9,91 \\
\hline \multirow{3}{*}{ Peso al nacer } & $<1500$ & 14 & 6,03 & 13 & 5,60 & 27 & 5,82 \\
\hline & $1500-2499$ & 88 & 37,93 & 97 & 41,81 & 185 & 39,87 \\
\hline & 2500-3999 & 130 & 56,04 & 122 & 52,59 & 252 & 54,31 \\
\hline \multirow{2}{*}{$\begin{array}{l}\text { Edad } \\
\text { gestacional }\end{array}$} & Prematuro & 124 & 53,45 & 123 & 53,01 & 247 & 53,23 \\
\hline & A término & 108 & 46,55 & 109 & 46,98 & 217 & 46,77 \\
\hline \multirow{3}{*}{$\begin{array}{l}\text { Peso } \\
\text { para edad } \\
\text { gestacional }\end{array}$} & Adecuado & 197 & 84,91 & 196 & 84,48 & 393 & 84,70 \\
\hline & Grande & 1 & 0,43 & 2 & 0,86 & 3 & 0,65 \\
\hline & Pequeño & 34 & 14,66 & 34 & 14,66 & 68 & 14,65 \\
\hline \multirow{3}{*}{$\begin{array}{l}\text { Apgar al } \\
\text { minuto }\end{array}$} & 0 a 3 & 9 & 3,90 & 6 & 2,60 & 15 & 3,20 \\
\hline & 4 a 6 & 8 & 3,40 & 13 & 5,60 & 21 & 4,50 \\
\hline & 7 a 10 & 215 & 92,70 & 213 & 91,80 & 428 & 92,20 \\
\hline \multirow{3}{*}{$\begin{array}{l}\text { Apgar a los } 5 \\
\text { minutos }\end{array}$} & 0 a 3 & 3 & 1,29 & 3 & 1,29 & 6 & 1,29 \\
\hline & 4 a 6 & 4 & 1,72 & 4 & 1,72 & 8 & 1,72 \\
\hline & 7 a 10 & 225 & 96,98 & 225 & 96,98 & 450 & 96,98 \\
\hline
\end{tabular}

En cuanto a la condición de egreso del recién nacido gemelo se encontró $4,95 \%$ de fallecimiento, siendo la mortalidad de $5,17 \%$ en los primeros gemelos y de $4,74 \%$ en el segundo gemelo (Tabla 3 ).

Tabla 3

Condición de egreso del recién nacido gemelar

\begin{tabular}{lcccccc}
\hline \multirow{2}{*}{ Egreso } & \multicolumn{2}{c}{ Gemelo 1 } & \multicolumn{2}{c}{ Gemelo 2 } & \multicolumn{2}{c}{ Total } \\
\cline { 2 - 7 } & $\mathrm{N} .^{\circ}$ & $\%$ & $\mathrm{~N} .{ }^{\circ}$ & $\%$ & $\mathrm{~N} .^{\circ}$ & $\%$ \\
\hline Con patología & 141 & 60,78 & 148 & 63,79 & 289 & 62,28 \\
Fallece & 12 & 5,17 & 11 & 4,74 & 23 & 4,95 \\
Sano & 79 & 34,05 & 73 & 31,47 & 152 & 32,76 \\
\hline Total & 232 & 100 & 232 & 100 & 464 & 100 \\
\hline
\end{tabular}

En la figura 2 evidenciamos las principales complicaciones neonatales de los recién nacidos gemelares, siendo las más prevalentes 45,3\% de bajo peso al nacer, 41,45 \% anemia, 18,16\% RCIU y $11,11 \%$ membrana hialina. 


\section{Figura 2}

Patología de recién nacidos gemelares según orden de nacimiento

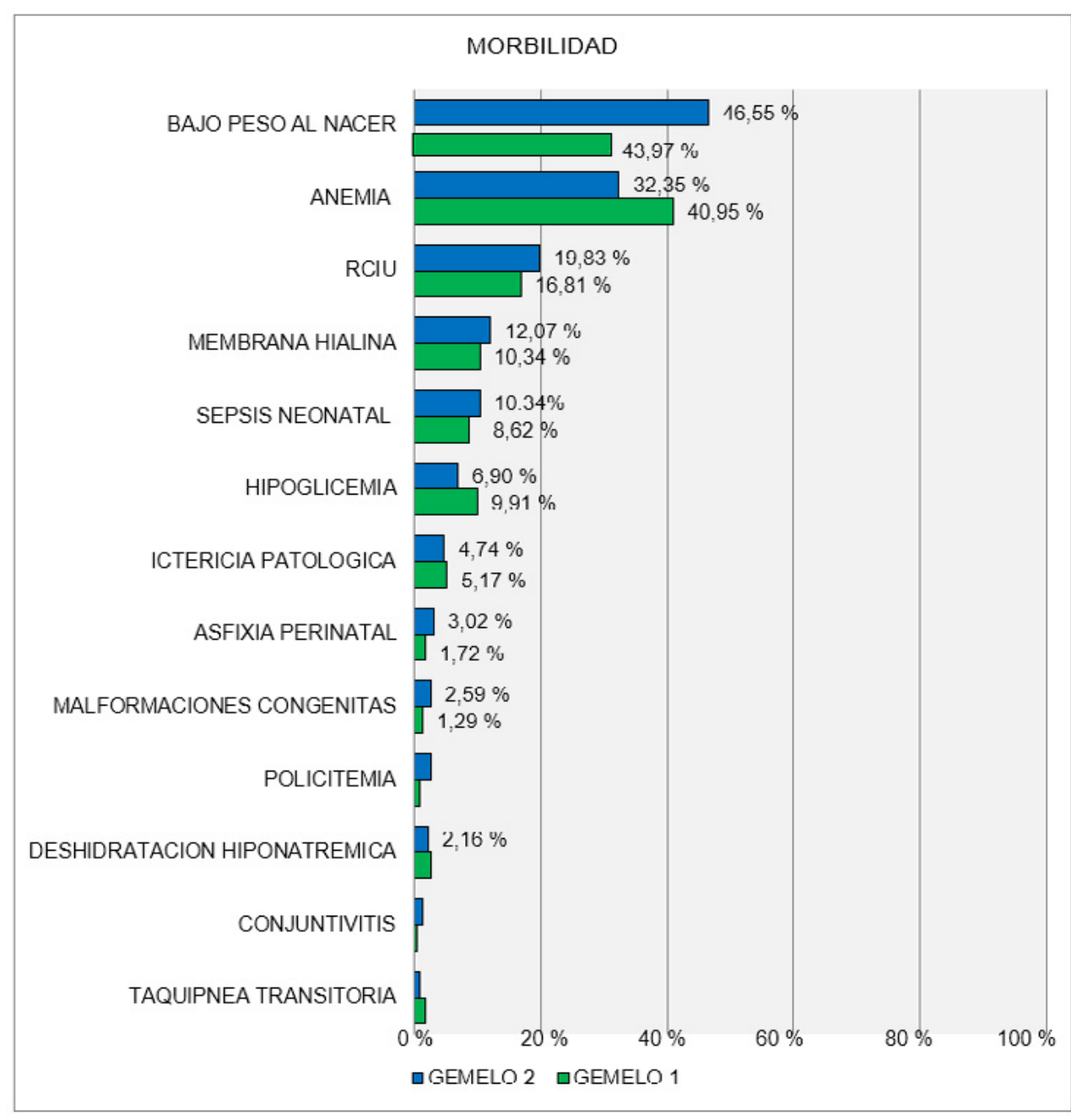

\section{Discusión}

Durante el periodo 2009 a 2018 en el Hospital Hipólito Unanue de Tacna, se atendieron 34 032 recién nacidos vivos, de los cuales 472 fueron gemelares; es decir, 1 de cada 72 nacidos vivos, siendo la incidencia de $1,39 \%$ o 13,9 por cada 1000 nacidos vivos. Esta cifra se encuentra dentro del rango reportado por Hernández en México en el 2008 quien describe la tasa de embarazos gemelares de 7,1 a 14,4 por 1000 nacimientos. ${ }^{6}$ Mientras que, en el Perú, en el Hospital Daniel A. Carrión, entre 2010 y 2014, la incidencia fue de 2,25 por 1000 nacidos vivos. $^{7}$

La incidencia fue inferior a lo descrito por Pacheco en diferentes países: Italia 1:86, Grecia 1:130, Japón 1:50, China 1:300; por Hernández de Cuba 1:96, entre los años 1991 a 2000; y Nazer en Chile 1:80, entre los años 2001 a 2004..$^{8-10}$ Estas diferencias entre los hallazgos y lo publicado por otros autores tendría como explicación a la existencia de factores intrínsecos y extrínsecos que influyen directamente en las tasas de incidencia del embarazo gemelar.

Durante los diez años del estudio, se ha observado un discreto incremento en la incidencia, semejante a lo encontrado por Fuster y Zoluaga en España, en el 2008, alcanzando 1,5\%. ${ }^{11}$ Mientras que en otros países describen cifras mucho menores como en Honduras $0,7 \% .{ }^{12}$ A ello ha contribuido la mayor demanda de las técnicas de reproducción asistida, esta situación refleja la evolución de la sociedad actual que genera una demanda de mayor atención y estudio de esta población. ${ }^{13}$

El $49,35 \%$ de gemelos fueron de sexo masculino y $50,65 \%$ de sexo femenino. Este predominio de sexo femenino sobre el masculino, también fue reportado por Cano, entre 2000 a 2010, en el mismo hospital de este estudio. ${ }^{14}$ De igual manera Cañas en Colombia, en el 2008, confirma que el sexo más frecuente fue el femenino con 51,4 $\% .{ }^{15}$ 
Tanto el primer como el segundo gemelo terminó en cesárea en el 90,09 \% y sólo 9,91 \% en parto espontáneo. Con respecto al parto espontáneo sus cifras son inferiores a lo reportado por Ramos en Cuba en el 2008 con $25 \%$ de parto vía vaginal. ${ }^{16}$ En contraparte Molina en un estudio en Bogotá Colombia reporta que la vía del parto más frecuente fue la cesárea en el $95 \% .^{17}$

Encontramos que el primer gemelo, 43,1 $\%$ tuvo bajo peso y el segundo gemelo, el $47,4 \%$. Los resultados son similares a lo publicado por Wen en Canadá quien encontró que $46,8 \%$ de gemelos nacieron con bajo peso. ${ }^{5}$ Además, evidenciamos que en el presente estudio el peso promedio del primer gemelo fue 2489 gramos y del segundo 2490 gramos. Estos resultados se encuentran por encima de lo obtenido por otros estudios, como el de González y Salvador en Lima, quienes reportaron que el peso promedio de los recién nacidos fue de 2293 gramos. ${ }^{18}$ Además, Cano reportó en un estudio realizado en el Hospital Hipólito Unanue Tacna, durante 2000 a 2010, que el promedio de peso para el primer gemelo fue $2511 \mathrm{~g}$ y para el segundo 2522 $\mathrm{g}$, valores superiores al encontrado en el estudio. ${ }^{14}$ Asimismo, Laterra en Argentina también describe pesos más bajos que los gemelos en el presente estudio (media=2240 g)19 y Cañas encontró un promedio de peso 2096 gramos. $^{15}$

La mayoría de los gemelos presentó adecuado peso para la edad gestacional $(84,1 \%$ primer gemelo y $84,48 \%$ segundo gemelo), sin mayores diferencias entre pequeños y grandes para la edad gestacional. Aunque no podemos comparar los resultados obtenidos, debido a que no fue estudiado en otras investigaciones, aportamos que no existe diferencia notoria en la relación peso para la edad gestacional, según el orden de nacimiento de los gemelos.

La mayoría de recién nacidos gemelos fueron prematuros, $53,5 \%$ el primer gemelo y $53 \%$ el segundo, la edad gestacional promedio fue de 35,5 semanas. Según la Sociedad Española de Pediatría, más del $50 \%$ de los gemelos son prematuros, valor que coincide con el estudio. ${ }^{20}$ Bedoya, en el Hospital Rebagliati encontró que $65,8 \%$ de prematuridad, con una media de 35,3 semanas y desviación estándar 2,29 semanas. ${ }^{1}$ Molina en Bogotá Colombia encontró tasas más altas de prematuridad $74,8 \%$, con un promedio de 34 semanas, cifra superior a este estudio, pero con un promedio para la edad gestacional menor a la presente investigación. ${ }^{17}$
El puntaje del Apgar es usado para la valoración de la adaptación al nacer. ${ }^{20}$ En este estudio en el primer gemelo 7,3\% presentó Apgar al minuto menor de $7, y$ en el segundo gemelo 8,2 $\%$. A los 5 minutos $3 \%$ de los primeros y segundos gemelos presentó Apgar a los 5 minutos menor de 7. Los resultados obtenidos difieren con lo reportado por Cano en estudio previo en el Hospital Hipólito Unanue, donde encontró que el 10,1\% presentó depresión al nacer, con mayor predominio en el segundo gemelo. ${ }^{14}$ Sin embargo, en los pacientes del presente estudio, la frecuencia de Apgar menor de 7 fue similar en el primer y segundo gemelo, tanto al minuto y cinco minutos de nacido. ${ }^{21}$

Respecto a la mortalidad, se encontró que el $4,95 \%$ de los gemelos fallecieron durante el periodo neonatal, siendo la mortalidad del primer gemelo $5,17 \%$ y del segundo $4,74 \%$. El conocimiento de la mortalidad neonatal es un hecho de gran importancia porque proporciona una imagen del nivel de desarrollo y calidad de salud. Esta cifra fue similar a lo reportado por Laterra quien evidencia que la mortalidad neonatal fue de 3,8 \%. ${ }^{19}$ Asimismo, Briceño-Pérez en Maracaibo Venezuela muestra una mortalidad más elevada de $18,3 \% .{ }^{22}$ Contrario a lo referido por Ramos en Camaguey Cuba, quien mostró que en su estudio no se registraron muertes neonatales. ${ }^{23}$

Respecto a las complicaciones neonatales de los gemelares, encontramos $45,3 \%$ de bajo peso al nacer, $41,5 \%$ anemia, $18,2 \%$ RCIU y 11,1 $\%$ membrana hialina. Briceño-Pérez encontró como principal complicación neonatal, $52,7 \%$ bajo peso al nacer, Zam13 en Cienfuegos Cuba, demuestra 50 $\%$ de bajo peso. ${ }^{22}$ Mientras que estudios realizados por Gonzales en el Hospital Cayetano Heredia de Lima evidencian $41,6 \%$ de RCIU mucho mayor a lo encontrado en este estudio. ${ }^{18}$ Tang refiere que la patología asociada en los neonatos gemelares ha sido predominantemente respiratoria, la membrana hialina, entre $6 \%$ y $29 \%$ de gemelos. 24 Mientras que Cañas en El Valle Colombia, afirma que las principales complicaciones fueron membrana hialina $(15,3 \%)$ y taquipnea transitoria $(8,6 \%)$, resultados que son diferentes a los hallazgos del presente estudio, siendo la membrana hialina 11,1 $\%$ ocupando el cuarto lugar y taquipnea transitoria $1,28 \% .^{15}$ 


\section{Conclusiones}

Se concluye que la incidencia de recién nacidos gemelares fue de 1,39 por cada 100 RNV, con una discreta tendencia ascendente en los últimos 10 años. Las características perinatales más frecuentes de los recién nacidos gemelares fueron $90,1 \%$ nacido por cesárea, bajo peso al nacer $45,7 \%$, pretérmino 53,2 \%, APGAR menor de 7 fue $7,7 \%$, y a los 5 minutos 3,01\%. Las complicaciones neonatales más frecuentes en los recién nacidos gemelares fueron: bajo peso al nacer (45,3\%), anemia $(41,5$ $\%)$, retardo en el crecimiento intrauterino $(18,2 \%)$, enfermedad de membrana hialina $(11,1 \%)$ y sepsis neonatal $(9,4 \%)$. La muerte neonatal representó el $4,95 \%$ del total de recién nacidos gemelos. De todas las complicaciones analizadas, no hemos podido evidenciar que existan diferencias importantes entre el primer y segundo gemelo.

Se recomienda dar alcance del presente trabajo a las entidades de salud que atienden partos gemelares, dando a conocer las complicaciones neonatales en los recién nacidos gemelares como: bajo peso al nacer, anemia, retardo en el crecimiento intrauterino, membrana hialina y sepsis neonatal encontrados en el presente estudio. $Y$ establecer un sistema de diagnóstico oportuno mediante el uso de ecografía en el primer trimestre y manejo especializado de los gemelos discordantes debido a que tienen mayor riesgo de complicaciones neonatales.

\section{Referencias}

1. Bedoya V, De La Cruz V, Correa L. Complicaciones fetales asociadas al parto en embarazos gemelares entre setiembre 2014 - setiembre 2015, Hospital Nacional Edgardo Rebagliati Martins. Tesis para optar el título profesional de Médico Cirujano. Universidad Ricardo Palma. Lima, 2016.

2. Refuerzo JS, Momirova V, Peaceman AM, Sciscione A, Rouse DJ, Caritis SN, et al. Neonatal outcomes in twin pregnancies delivered moderately preterm, late preterm, and term. Am J Perinatol. 2010; 27: 537-42.

3. Blondel B, Kogan MD, Alexander GR, Dattani N, Kramer MS, Macfarlane A, et al. The impact of the increasing number of multiple births on the rates of preterm birth and low birth weight: an international study. Am J Public Health. 2002; 92: 1323-30.

4.Monset-Couchard M, De Bethmann O, Relier JP.
Long term outcome of small versus appropiate size for gestational age co-twins/triplets. Arch Dis Child Fetal Neonatal Ed. 2004; 89: F310-4.

5. Wen SW, Fung KF, Huang L, Demissie K, Joseph KS, Allen $A C$ et al. Fetal and neonatal mortality among twin gestations in Canadian population: the effect of intrapair birthweight discordance. Am J Perinatol. 2005; 22: 279-86.

6. Hernández HR, Ochoa TM, Flores SR, Cortés FR, Forsbasch SG. Prevalencia de embarazos múltiples: incremento en la última década. Gineco Obstet Mex 2008; 76:507 -11.

7. Lacunza PR, Ávalos GJ, Bueno BE, Julcamoro AM. Implicancias epidemiológicas de la gestación múltiple. Simposio Embarazo Múltiple. Hospital Nacional Daniel A. Carrión. Mayo 2015.

8. Pacheco J. Ginecología Obstetricia y Reproducción. Segunda Edición. Editorial REP. 2007 Pág. 13051319

9. Hernández $\mathrm{C}$, Hernández $\mathrm{H}$. Embarazos Múltiples y su influencia en los principales indicadores perinatales. Revista Cubana de Obstetricia 2003; 29.

10. Nazer J. Águila A. Cifuentes L. La frecuencia de nacimiento de gemelos aumentó en un hospital chileno coincidiendo con el consumo periconcepcional de harina fortificada con ácido fálico. Rev Med Chile 2006; 134: 48-52.

11. Fuster V, Zuluaga P, Colantonio S, De Bias C. Factors associated with recent increase of multiple births in Spain. Twin Research and Human Genetics 2008; 11: 70-76.

12. Muñoz O, Valladares $C$. Embarazo gemelar como factor de riesgo materno en el hospital Escuela Tegucigalpa, Honduras. Rev Med Post UNAH 2001; 6: 262-266.

13. Zam M, Fernández A, Jiménez González G, Díaz Brito A, Torres González C. Caracterización perinatal del embarazo gemelar en Cienfuegos 2001-2002. Revista Electrónica de las Ciencias Médicas en Cienfuegos. Cuba. Medisur 2004; 2.

14. Cano UA. Incidencia y complicaciones de los recién nacidos gemelares en el Hospital Hipólito Unanue de Tacna 2000-2010. Tesis para optar el título profesional de Médico Cirujano. Universidad 
Nacional Jorge Basadre Grohmann, Facultad de Ciencias de la Salud. Tacna, Perú. 2012.

15. Cañas G, Cifuentes B. Morbimortalidad del embarazo gemelar en el hospital Universitario del Valle. Revista de Obstetricia y Ginecología. Colombia 2008;1:15-18. Disponible en: http://www. encolombia.com/obstetricia50399contenido.htm

16. Ramos PE. Sánchez LM, Caridad IA. Rodríguez MM Comportamiento del Embarazo Múltiple y sus principales indicadores perinatales. Camaguey. Cuba 2008; 2.

17. Molina GS, Araméndiz JM, Beltrán AS, Rojas AJ, Acuña OE, Solano MA. Resultado perinatal de embarazos gemelares atendidos en dos instituciones de alta complejidad en Bogotá, Colombia. Revista Chilena Obstetricia y Ginecología 2015; 80(1): 18-23.

18. Gonzales Del Riego M, Salvador J. Embarazo múltiple. Estudio en el Hospital Nacional Cayetano Heredia. Ginecología y obstetricia/ Sociedad Peruana de Obstetricia y Ginecología. 1997. Ginecol \& Obstet (Perú) 1997; 43(3).

19. Laterra C, Susacasa S, Frailuna M, Flores L, Secondi V. Embarazo doble: diez años de resultados perinatales en el Hospital Materno Infantil Ramón Sardá (1992-2001). Revista del Hospital Materno Infantil Ramón Sardá, Buenos Aires, Argentina. Rev Hosp Mat Inf Ramón Sardá 2006; 25.
20. Rellan RR, García RC, Paz AG. Recién nacido prematuro. Protocolos Diagnósticos Terapéuticos de la AEP: Neonatología. 2008.

21. Briceño G. Morbimortalidad del segundo gemelar estudio comparativo entre cesárea y parto vaginal. Hospital Central Antonio María Pineda Barquisimeto, enero 1991- diciembre 1992. Boletín Médico de Postgrado. Venezuela.

22. Briceño-Pérez C, Briceño-Sanabria L. Morbimortalidad fetal y neonatal en embarazo gemelar. Hospital Chiquinquirá de Maracaibo. 1991-2000. Rev Obstet Gineco Venez. 2005; 65: 1-8.

23. Ramos PE, Sánchez LM, Amador de Varona C, Rodríguez M. Comportamiento del embarazo múltiple y sus principales indicadores perinatales. Camaguey Cuba. 2004.

24. Tang L, Mere J. Embarazo Múltiple. Revista Ginecología y Obstetricia 1996; 42 (3).

\section{Correspondencia:}

vitor_ele@hotmail.com
Fecha de recepción: 15/04/2021

Fecha de aceptación: 05/08/2021 